OPEN ACCESS

Edited by:

Elmar Laistler

Medical University of Vienna, Austria

Reviewed by:

Andrew Webb,

Leiden University, Netherlands

Arthur W. Magill,

German Cancer Research Center

(DKFZ), Germany

${ }^{*}$ Correspondence:

Daniel Wenz

daniel.wenz@epfl.ch

Specialty section:

This article was submitted to

Medical Physics and Imaging,

a section of the journal

Frontiers in Physics

Received: 03 March 2021

Accepted: 10 May 2021

Published: 15 June 2021

Citation:

Wenz D and Gruetter R (2021) DipoleFed Rectangular Dielectric Resonator

Antennas for Magnetic Resonance Imaging at 7 T: The Impact of QuasiTransverse Electric Modes on Transmit

Field Distribution.

Front. Phys. 9:675509.

doi: 10.3389/fphy.2021.675509

\section{Dipole-Fed Rectangular Dielectric Resonator Antennas for Magnetic Resonance Imaging at 7T: The Impact of Quasi-Transverse Electric Modes on Transmit Field Distribution}

\author{
Daniel Wenz ${ }^{1,2 *}$ and Rolf Gruetter ${ }^{3}$ \\ ${ }^{1}$ CIBM Center for Biomedical Imaging, Lausanne, Switzerland, ${ }^{2}$ Animal Imaging and Technology, Ecole Polytechnique Federale \\ de Lausanne (EPFL), Lausanne, Switzerland, ${ }^{3}$ Laboratory of Functional and Metabolic Imaging (LIFMET), Ecole Polytechnique \\ Federale de Lausanne (EPFL), Lausanne, Switzerland
}

Shortened dipole antennas based on rectangular dielectric blocks play an important role in ultrahigh field magnetic resonance imaging (UHF-MRI) radio frequency (RF) coil design. However, the generally assumed direct contact with the subject is difficult to maintain in typical in vivo settings. We have previously observed that certain dielectrically shortened dipole antennas can produce a substantially altered transmit field distribution with a very low transmit efficiency when the block and the sample are physically separated. Therefore, the aim of this study was to determine a) why certain designs of dielectrically shortened dipole antennas can produce an inefficient transmit field when the block and the sample are physically separated and b) how this depends on key parameters such as rectangular block geometry, dielectric constant, loading geometry, and RF feeding. In this work, two main types of quasi-transverse dielectric modes were found in different rectangular block geometries and interpreted as $T E_{11 \delta}^{z}$ (MR efficient) and $T E_{1 \delta \delta}^{y}$ (MR inefficient), and their impact on in vivo MRI experiments involving the human head, calf, and wrist was explored. This study shows, for the first time, why certain antennas preserve their transmit field efficiency despite physical separation from the sample. We conclude that the proposed approach has the potential to provide new insights into dipole antenna design for UHF-MRI.

Keywords: dielectric resonator antenna, dipole antenna, dielectric mode, radio frequency coil, ultrahigh field magnetic resonance imaging, 7 Tesla

\section{INTRODUCTION}

Ultrahigh field magnetic resonance imaging (UHF-MRI) in humans (magnetic field strength $\mathrm{B}_{0} \geqq 7 \mathrm{~T}$ ) can be challenging due to the short wavelength (about $12 \mathrm{~cm}$ ) and increased radio frequency (RF) power deposition in the tissue [1]. Multichannel RF coil arrays, which are widely used to address these issues, allow for transmit field $\left(\mathrm{B}_{1}{ }^{+}\right)$shimming to optimize the $\mathrm{B}_{1}{ }^{+}$homogeneity, thereby providing significant signal-to-noise ratio (SNR) gains and higher acceleration factors in parallel MRI [2-5]. Such multichannel arrays are typically built with loop elements. However, in recent years, it has been shown that dipole antennas can lead to significant $\mathrm{B}_{1}{ }^{+}$efficiency gains in deeper anatomical regions [6-9], and they also support curl-free current patterns that are crucial to 
approach the ultimate intrinsic SNR in UHF-MRI [10]. Increasing the number of channels in dipole antenna arrays requires modification to the single dipole antenna element geometry because the length of dipole antennas in free space $(\sim 50 \mathrm{~cm}$ at $297.2 \mathrm{MHz})$ is not suitable for most in vivo MR applications.

Since the wavelength is inversely proportional to the square root of dielectric permittivity $\varepsilon_{\mathrm{r}}$, one promising approach to shorten dipole antennas is to use a high $\varepsilon_{\mathrm{r}}$ medium [6, 11-13]. Unfortunately, the dimensions of dielectric blocks used in previous investigations were rather large, which made it difficult to use them as building blocks in very large dipole antenna arrays (e.g., 32, 64, or 128 elements). Most of the studies followed what Raaijmakers et al. suggested in their study [6], that is, the height should be at least $1 / 4$ of wavelength $\lambda$. Later, Ipek et al. [14] found that the optimal transmit field efficiency can be achieved for a block with $150 \mathrm{~mm} \times 50 \mathrm{~mm} \times 50 \mathrm{~mm}$ and $\varepsilon_{\mathrm{r}}$ between 90 and 110 . Recently, Eigentler et al. [15] developed a self-grounded bowtie antenna, which was immersed in a small volume filled with deuterium oxide $\left(\mathrm{D}_{2} \mathrm{O}\right)$, but they used quite a large water bolus to ensure a direct contact with a cuboid phantom.

All these reports $[6,13-15]$ assumed there was a direct contact between the dielectric block and the human body (or a phantom with a flat surface). Yet, such a contact is rather difficult to achieve for a solid, rectangular geometry, and it may not always be feasible in clinical settings. The curvature of the human head, for example, makes meeting this condition particularly difficult. Therefore, it is reasonable to assume that the dielectric block and various anatomical structures are physically separated. A detailed study, dedicated to this particular, realistic scenario, has not been conducted yet.

In a previous study, we observed that certain types of dielectrically shortened dipole antennas produced an efficient transmit field in the presence of a small air gap, while others did not [16]. We hypothesized that different dielectric modes can be induced within the rectangular block, thereby affecting the antenna performance; if a rectangular dielectric block is sufficiently large, dielectrically shortened dipole antennas can be interpreted as dipole-fed rectangular dielectric resonator antennas. Rectangular dielectric resonator antennas can be characterized by quasi-transverse electric modes [17-20], and UHF-MRI can be an excellent tool to visualize them if water is used as the dielectric medium [21, 22].

Therefore, the aim of this study was to determine: a) why certain dipole-fed dielectric resonator antennas preserve (and others do not) the transmit field distribution and efficiency when the block and the object are physically separated, b) which parameters play a critical role in this context, and c) to what extent different quasi-transverse electric modes can influence in vivo human MRI at $7 \mathrm{~T}$.

\section{METHODS}

Numerical electromagnetic field and specific absorption rate (SAR) simulations were performed using the finite-difference

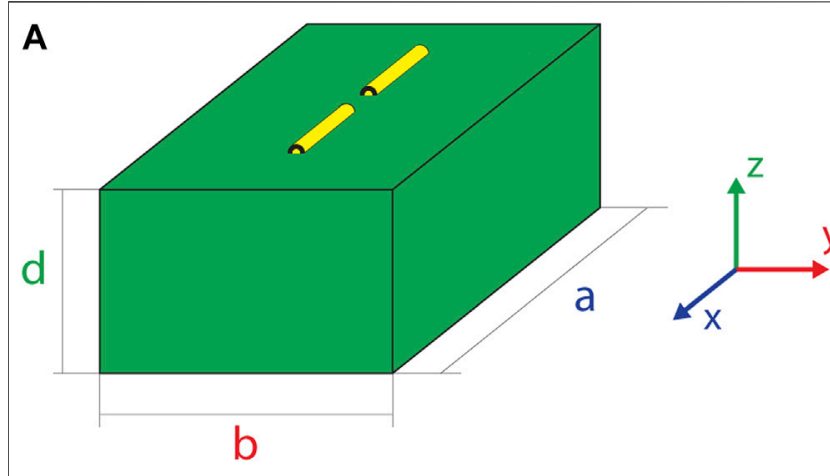

B

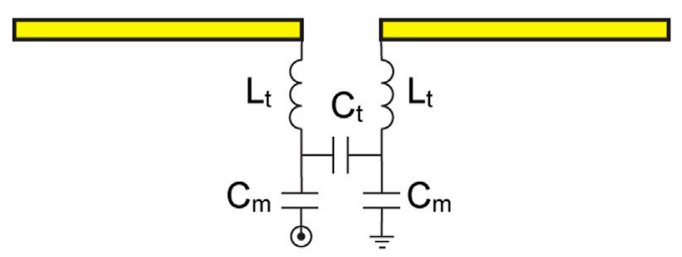

FIGURE 1 | (A) The frame of reference used to calculate the cutoff frequency and to determine the type of the dielectric mode that propagates across dielectric blocks. The following transformation from this system to the system of coordinates in MRI environment would have to be made: $x=z$, $y=x, z=y)$. (B) Matching network diagram.

time-domain solver of Sim4Life (Sim4Life, Zurich, Switzerland). Copper elements were modeled as perfect electrical conductors. The excitation signal was of Gaussian type (center frequency = 297.2 MHz and bandwidth $=300 \mathrm{MHz}$ ). The grid was manually adjusted for all the components in the simulation. For conductors, dielectric blocks and ports, the smallest mesh cell was $2 \mathrm{~mm}$ ( $1 \mathrm{~mm}$ when the effect of the block/phantom physical separation was investigated; $0.2 \mathrm{~mm}$ for $0.5 \mathrm{~mm}$ gap for $\varepsilon_{\mathrm{r}}=200$ ), while for the phantoms it was $4 \mathrm{~mm}$. The RF shield in the magnet and polymethylmethacrylate (PMMA) boxes were not included in the simulations. Two types of phantoms were used in the electromagnetic field simulations: a spherical one, which mimics the human head (radius $=85 \mathrm{~mm}, \varepsilon_{\mathrm{r}}=50.6, \sigma=0.66 \mathrm{~S} / \mathrm{m}$ ), and a cuboid one $\left(300 \mathrm{~mm} \times 300 \mathrm{~mm} \times 300 \mathrm{~mm}, \varepsilon_{\mathrm{r}}=34, \sigma=0.47 \mathrm{~S} / \mathrm{m}\right)$, which is more relevant for body applications, with dimensions identical to those used in previous studies $[6,13]$. Moreover, both phantoms represent two different levels of curvature: a flat (cuboid) and a rounded one (spherical). For all the elements in the work, we used the transmit field efficiency defined as $B_{1}^{+} / \sqrt{P}$, where $P$ is the input power, and the SAR efficiency defined as $B_{1}^{+} / \sqrt{S A R_{10 g}}$, where $S A R_{10 g}$ is the maximum SAR averaged over $10 \mathrm{~g}$. The simulation results obtained using Sim4Life were normalized to $1 \mathrm{~W}$ input power.

The transmit field distribution within the spherical phantom was studied for different rectangular block geometries and different values of dielectric permittivity $\left(\varepsilon_{\mathrm{r}}=35,50,80,100\right.$, $150,200,300$, and 500), assuming a $5-\mathrm{mm}$ air gap between the block and the phantom. A constant conductivity value, close to 
TABLE 1 | The dimensions of rectangular blocks (in millimeters) used in simulations from Figure 2. The dimension a ( $\left.a_{0}\right)$ was constant for each $\varepsilon_{\mathrm{r}}$ value: 35 (242 mm), 50 (202 mm), 80 (160 mm), 100 (144 mm), 150 (118 mm), 200 (102 mm), 300 (84 mm), and 500 (64 mm). Four variations of $b\left(b_{0}, 0.75,0.5 b_{0}\right.$, and $\left.0.25 b_{0}\right)$ and for each $b$ four $d / b(0.75,0.5,0.25$, and 0.125$)$ ratios were investigated.

\begin{tabular}{|c|c|c|c|c|c|c|c|c|c|}
\hline \multirow[t]{2}{*}{$b$} & \multirow[t]{2}{*}{$d / b$} & \multicolumn{8}{|c|}{ Dielectric permittivity $\varepsilon_{\mathrm{r}}$} \\
\hline & & 35 & 50 & 80 & 100 & 150 & 200 & 300 & 500 \\
\hline \multirow[t]{4}{*}{$b=b_{0}$} & 0.75 & $121 / 90.7$ & $101 / 75.7$ & $80 / 60$ & $72 / 54$ & $59 / 44.25$ & $51 / 38.2$ & $42 / 31.5$ & $32 / 24$ \\
\hline & 0.5 & $121 / 60.5$ & $101 / 50.5$ & $80 / 40$ & $72 / 36$ & $59 / 29.5$ & $51 / 25.5$ & $42 / 21$ & $32 / 16$ \\
\hline & 0.25 & $121 / 30.2$ & $101 / 25.2$ & $80 / 20$ & $72 / 18$ & $59 / 14.75$ & $51 / 12.7$ & $42 / 10.5$ & $32 / 8$ \\
\hline & 0.125 & $121 / 15.1$ & $101 / 12.1$ & $80 / 10$ & $72 / 9$ & $59 / 7.37$ & $51 / 6.3$ & $42 / 5.25$ & $32 / 4$ \\
\hline \multirow[t]{4}{*}{$b=0.75 b_{0}$} & 0.75 & $90.7 / 68$ & $75.7 / 56.8$ & $60 / 45$ & $54 / 40.5$ & $44.2 / 33.1$ & $38.2 / 28.6$ & $31.5 / 23.6$ & $24 / 18$ \\
\hline & 0.5 & $90.7 / 45.3$ & $75.7 / 37.5$ & $60 / 30$ & $54 / 27$ & $44.2 / 22.1$ & $38.2 / 19.1$ & $31.5 / 5.7$ & $24 / 12$ \\
\hline & 0.25 & $90.7 / 22.6$ & $75.7 / 18.75$ & $60 / 15$ & $54 / 13.5$ & $44 . .2 / 11$ & $38.2 / 9.5$ & $31.5 / 7.87$ & $24 / 6$ \\
\hline & 0.125 & $90.7 / 11.3$ & $75.7 / 9.3$ & $60 / 7.5$ & $54 / 6.75$ & $44.2 / 5.5$ & $38.2 / 4.7$ & $31.5 / 3.93$ & $24 / 3$ \\
\hline \multirow[t]{4}{*}{$b=0.5 b_{0}$} & 0.75 & $60.5 / 45.3$ & $50.5 / 37.8$ & $40 / 30$ & $36 / 27$ & 29.5/22.1 & 25.5/19.1 & 21/15.7 & $16 / 12$ \\
\hline & 0.5 & $60.5 / 30.2$ & $50.5 / 25.2$ & $40 / 20$ & $36 / 18$ & 29.5/14.7 & $25.5 / 12.7$ & 21/10.5 & $16 / 8$ \\
\hline & 0.25 & $60.5 / 15.1$ & $50.5 / 12.6$ & $40 / 10$ & $36 / 9$ & $29.5 / 7.3$ & 25.5/6.3 & $21 / 5.2$ & $16 / 4$ \\
\hline & 0.125 & $60.5 / 7.5$ & $50.5 / 6.3$ & $40 / 5$ & $36 / 4.5$ & 29.5/3.6 & 25.5/3.1 & $21 / 2.6$ & $16 / 2$ \\
\hline \multirow[t]{4}{*}{$b=0.25 b_{0}$} & 0.75 & $30.2 / 22.6$ & $25.25 / 18.9$ & $20 / 15$ & 18/13.5 & $14.7 / 11$ & $12.7 / 9.5$ & $10.5 / 7.8$ & $8 / 6$ \\
\hline & 0.5 & $30.2 / 15.1$ & $25.25 / 12.6$ & $20 / 10$ & $18 / 9$ & $14.7 / 7.3$ & $12.7 / 6.3$ & $10.5 / 5.2$ & $8 / 4$ \\
\hline & 0.25 & $30.2 / 7.5$ & $25.25 / 6.3$ & $20 / 5$ & $18 / 4.5$ & $14.7 / 3.6$ & 12.7/3.1 & $10.5 / 2.6$ & $8 / 2$ \\
\hline & 0.125 & $30.2 / 3.7$ & $25.25 / 3.1$ & $20 / 2.5$ & $18 / 2.25$ & $14.7 / 1.8$ & $12.7 / 1.6$ & $10.5 / 1.3$ & $8 / 1$ \\
\hline
\end{tabular}

the one for $\mathrm{D}_{2} \mathrm{O}(\sigma=0.065 \mathrm{~S} / \mathrm{m})[11]$, was used for all the blocks explored in this work. To identify the dielectric modes excited in a dipole-fed rectangular antenna, we used a coordinate system consistent with the commonly used convention (Figure 1). Note that in this work, we refer to the frame of reference from Figure $\mathbf{1}$ and not to the one typically used in MRI. Converting from the antenna frame of reference to the one for MRI requires the following transformation: $\mathrm{x} \rightarrow \mathrm{z}, \mathrm{y} \rightarrow \mathrm{x}$, and $\mathrm{z} \rightarrow \mathrm{y}$.

Each dielectric mode was described by three subscripts: $m, n$, and $l$. Additionally, one superscript ( $\mathrm{x}, \mathrm{y}$, or $\mathrm{z}$ ) was used to indicate the direction of propagation for a given quasi-transverse electric (TE) mode. The fraction $\delta$ means that the given index was equal to 0 (see [19]). The geometry of each rectangular block and each $\varepsilon_{\mathrm{r}}$ value was determined as follows: first, we found the dimensions of the smallest possible square geometry for which the $T E_{11 \delta}$ mode was allowed to propagate according to the following formula [19]:

$$
f_{\text {cutoff }}=\frac{c}{2 \pi \sqrt{\epsilon_{r}}} \sqrt{\left(\frac{\pi m}{a}\right)^{2}+\left(\frac{\pi n}{b}\right)^{2}+\left(\frac{\pi l}{d}\right)^{2}}
$$

where $c$ is the velocity of light; $m, n$, and $l$ are the dielectric mode indices ( $\delta$ means that there is a fraction of a field half-cycle in the given direction); and $a, b$, and $d$ are the block dimensions according to the coordinate system in Figure 1. For instance, to calculate the cutoff frequency using Eq. 1 for $T E_{11 \delta}$ and $T E_{1 \delta \delta}$ modes, the indices $m l n$ would be defined as 110 and 100 , respectively. Note that if $l=0$ (or if $l=0$ and $n=0$ ), Eq. 1 corresponds to the calculation of waveguide modes $T E_{11}$ (or $T E_{10}$ ) rather than the $3 \mathrm{D}$ resonance $T E_{111}$. Next, dimension $a$, which was critical to ensure that $T E_{11 \delta}$ was allowed to propagate, was multiplied by a factor of 2 . By doing so, we obtained initial values of $a$ and $b\left(a_{0}\right.$ and $\left.b_{0}\right)$, which were different for each $\varepsilon_{\mathrm{r}}$ (Table 1). The dimension $a\left(a_{0}\right)$ was constant for each $\varepsilon_{\mathrm{r}}$ value: 35
$(242 \mathrm{~mm}), 50(202 \mathrm{~mm}), 80(160 \mathrm{~mm}), 100(144 \mathrm{~mm}), 150$ $(118 \mathrm{~mm}), 200(102 \mathrm{~mm}), 300(84 \mathrm{~mm})$, and $500(64 \mathrm{~mm})$. Then, dimension $b$ was varied as follows: $b_{0}, 0.75 b_{0}, 0.5 b_{0}$, and $0.25 b_{0}$. For these dimensions of $b$, we investigated the following $d /$ $b$ ratios: $d / b=1,0.75,0.25$, and 0.125 . According to Eq. 1, for $b=b_{0}$ and $b=0.75 b_{0}$, the cutoff frequency for $T E_{21 \delta}$ was lower than the resonance frequency. However, the $T E_{21 \delta}$ mode was not observed in any of the investigated blocks regardless of the $\varepsilon_{\mathrm{r}}$ value. For each $\varepsilon_{\mathrm{r}}$ value, the relative wavelength was calculated $\left(\lambda_{r} \sim 1 / \sqrt{\varepsilon_{r}}\right)$, and the length of each dipole antenna was accordingly chosen: $42,35,28,25,20,18,14,11$, and $8 \mathrm{~mm}$ per dipole arm (5-mm distance between both arms) for $\varepsilon_{\mathrm{r}}$ from 35 to 500 . Half of the dipole antenna (copper wire, radius $=1 \mathrm{~mm}$ ) was immersed in the dielectric medium, while the other half was in contact with air (Figure 1). Each antenna was tuned to 297.2 MHz and matched to $50 \Omega$ using an LCC tuning/ matching network (L-series, C-parallel, C-series).

The transmit field efficiency in the spherical phantom could not be compared between all the blocks from Table $\mathbf{1}$ in a fair manner because higher $\varepsilon_{\mathrm{r}}$ values resulted in smaller blocks and shorter dipole antennas. For this purpose, a constant block geometry $(a=160 \mathrm{~mm}, b=60 \mathrm{~mm}$, and $d=7.5 \mathrm{~mm})$ and dipole antenna length ( $28 \mathrm{~mm}$ per arm) was defined for all analyzed $\varepsilon_{\mathrm{r}}$ values. The dimensions were chosen so that such a block could be used as one of the elements in a close-fitting 8channel transmit/receive array for brain imaging [11].

The effect of an air gap on the transmit field distribution in a cuboid phantom was studied for one larger block $\left(0.75 b_{0}, d /\right.$ $b=0.75$ ) for each $\varepsilon_{\mathrm{r}}$ value. The cuboid phantom was chosen for this purpose because it provided good coupling (flat surface) to the rectangular dielectric block; the transmit field distribution in the cuboid phantom was also benchmarked against the results obtained for the spherical phantom (rounded surface). Five different air gaps were studied: 1, 2, 3, 4, and 
$5 \mathrm{~mm}$ for all $\varepsilon_{\mathrm{r}}$ values. These simulations were extended by investigating the y-component of the magnetic field $\vec{H}\left(H_{y}\right)$ for one larger air gap $(20 \mathrm{~mm})$ for two low- $\varepsilon_{\mathrm{r}}$ blocks $(35,50)$ and one smaller air gap $(0.5 \mathrm{~mm})$ for one high- $\varepsilon_{\mathrm{r}}$ block (200). These results were compared to the ones obtained for the spherical phantom (5-mm air gap). The reason why the $\mathrm{H}$-field was considered instead of the B-field was to refer to the previous study conducted by Ipek et al. [14], which focused on the case when there is a perfect direct contact between the block and a large cuboid phantom.

Plane wave simulations were conducted for six different wave number and electric field vector configurations with the following parameters: number of mesh cells $=681,000$, excitation signal $=$ Gaussian, center frequency $=$ 297.2 MHz, and bandwidth $=300 \mathrm{MHz}$, amplitude $=1 \mathrm{~V}$. The dielectric block $(160 \mathrm{~mm} \times 70 \mathrm{~mm} \times 52.5 \mathrm{~mm})$ was defined as the wave source according to the approach provided by Sim4Life, which is based on total-field/ scattered-field (TF/SF) formulation (also called the planewave injector). In this method, only a certain region of the calculation domain, the total field region (the dielectric block in our case), propagates the plane wave.

Two dielectrically shortened dipole antennas $\left(\varepsilon_{\mathrm{r}}=80\right)$ were designed, built, and evaluated in MR experiments: $160 \mathrm{~mm} \times$ $70 \mathrm{~mm} \times 52.5 \mathrm{~mm}\left(0.75 b_{0}, d / b=0.75\right)$ and $160 \mathrm{~mm} \times 70 \mathrm{~mm} \times$ $17.5 \mathrm{~mm}\left(0.75 b_{0}, d / b=0.25\right)$. The prototypes were built of a PMMA shell (wall thickness $=3 \mathrm{~mm}$ ). The geometries were chosen so that different dielectric modes can be excited within the blocks. The reason for using $\varepsilon_{\mathrm{r}}=80$ was that water has an $\varepsilon_{\mathrm{r}}$ value close to 80 at $297.2 \mathrm{MHz}$. Water is readily available and easily imaged by MRI [22]. To visualize the dielectric modes within the different block geometries, deionized water was used, and the dipole antenna was tuned to $297.2 \mathrm{MHz}$ and matched to $50 \Omega$.

MR experiments were conducted with a 7.0-T $68-\mathrm{cm}$ bore scanner (Magnetom, Siemens Healthineers, Erlangen, Germany). A shielded cable trap, consisting of a capacitor soldered to the shield of the coaxial cable, was connected to each element and used in every experiment. MRI experiments were performed in one male subject (age $=29$ years, BMI = $28.5 \mathrm{~kg} / \mathrm{m}^{2}$ ), who had signed written consent approved by the local ethics committee, in three different regions of interest: head, calf, and wrist. It was not feasible to conduct additional experiments for other anatomical structures because the scanner used is dedicated to the human head. The goal was to investigate how different dielectric modes could propagate across various anatomical structures (with different loading geometries), given our prior knowledge on the differences between cuboid and spherical phantoms [16]. To visualize the magnetic field distribution within both blocks, standard gradient echo (GRE) imaging was used with the following parameters: repetition time $(\mathrm{TR})=8.6 \mathrm{~ms}$, echo time $(\mathrm{TE})=$ $4.0 \mathrm{~ms}$, field of view $(\mathrm{FOV})=250 \times 250 \mathrm{~mm}^{2}$, slice thickness $=$ $7.0 \mathrm{~mm}$, number of averages $=2, \mathrm{FA}=15^{\circ}$, and reference transmit voltage $=5 \mathrm{~V}$. For in vivo experiments, deionized water, which produced a very-high-intensity signal, compromising the in vivo image quality, was replaced by

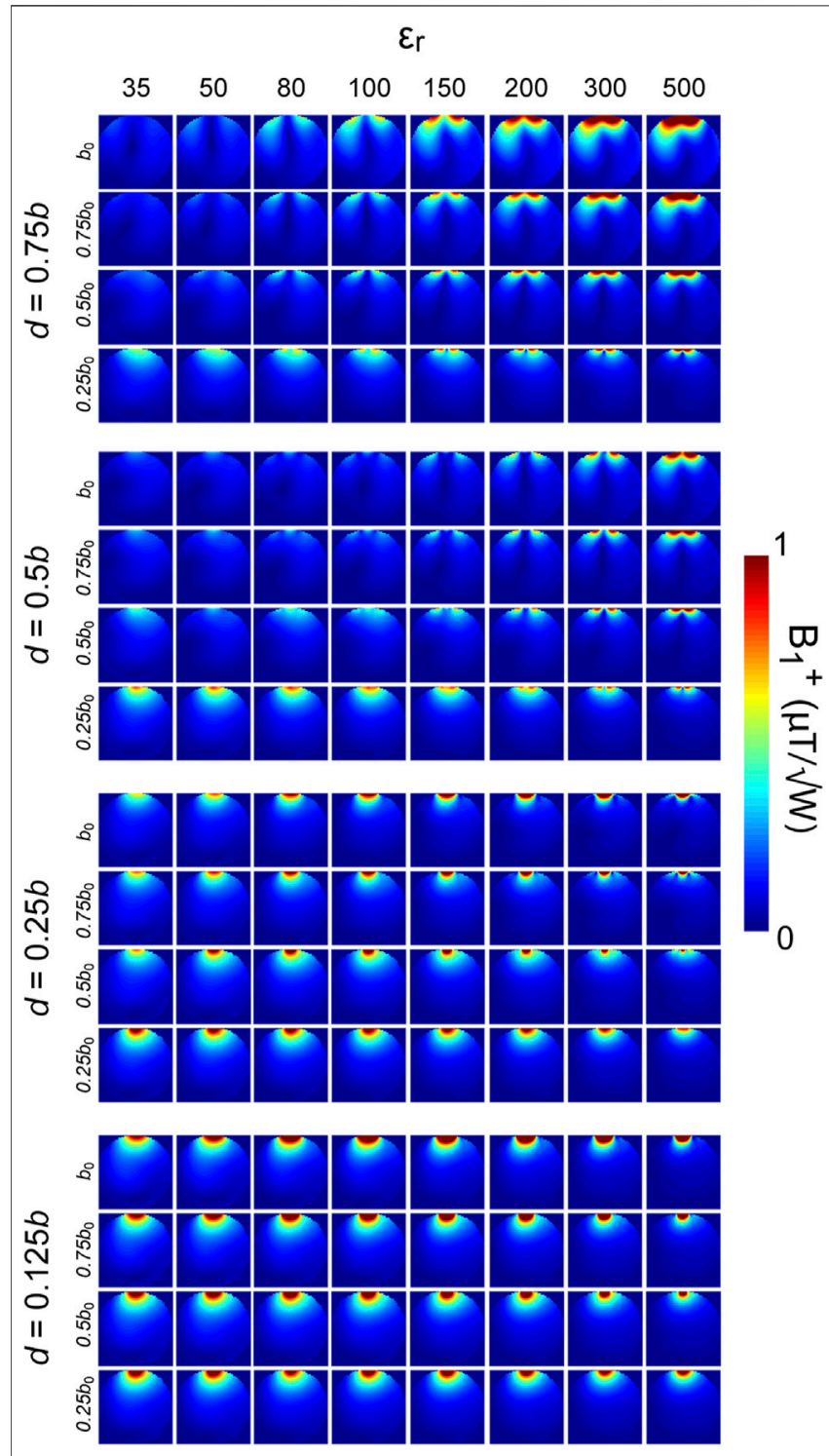

FIGURE 2 | Transmit field distribution in the spherical phantom for various geometries (Table 1) of dipole-fed rectangular dielectric resonator antennas and dielectric permittivity $\varepsilon_{\mathrm{r}}(35,50,80,100,150,200,300$, and 500). The distance between the bottom of the block and the phantom was $5 \mathrm{~mm}$. Each column depicts different $\varepsilon_{\mathrm{r}}$ values. The figure was divided into four groups (starting from the top): $d / b=0.75,0.5,0.25$, and 0.125 . Each group consists of four rows: $b=b_{0}, 0.75 b_{0}, 0.5 b_{0}$, and $0.25 b_{0}$. Substantial differences in the transmit field pattern and efficiency can be observed between different geometries for a given $\varepsilon_{r}$, as well as for the same $a / b / d$ ratio, but different $\varepsilon_{\mathrm{r}}$ values.

heavy water $\left(\mathrm{D}_{2} \mathrm{O}\right.$, Sigma Aldrich, Germany), and a 2-mm acrylonitrile butadiene styrene board was placed between the block and the subject. In vivo images were acquired by $3 \mathrm{D}-\mathrm{GRE}$ imaging with the following parameters: TR/TE $=6.5 / 2.82 \mathrm{~ms}$, FOV $=256 \times 240 \mathrm{~mm}^{2}$, slice thickness $=1.0 \mathrm{~mm}$, number of averages $=1, \mathrm{FA}=4^{\circ}$, and reference transmit voltage $=100 \mathrm{~V}$. The acquisition parameters of the RF pulse sequence were used to scan each body part. 
TABLE 2 | Maximum local SAR ${ }_{10 g}$ values in $W / k g$ provided for the simulations from Figure 2. The exact dimensions of the blocks can be found in Table 1. In general, for lower $b$ values $\left(0.5 b_{0}\right.$ and $\left.0.25 b_{0}\right)$, the thicker blocks yielded lower was the $\mathrm{SAR}_{10 \mathrm{~g}}$ than their thinner counterparts. This trend changed for $d / b=0.75$ for higher- $\varepsilon_{\mathrm{r}}$ blocks (300 and 500). For $b=b_{0}$, $S A R_{10 g}$ values for higher- $\varepsilon_{\mathrm{r}}$ blocks $(200,300$, and 500$)$ were found to be significantly higher for $d / b=0.75$ than for $d / b=0.125$.

\begin{tabular}{lccccccccc}
$\boldsymbol{b}$ & $\boldsymbol{d} / \boldsymbol{b}$ & \multicolumn{8}{c}{ Dielectric permittivity $\boldsymbol{\varepsilon}_{\mathbf{r}}$} \\
\cline { 3 - 10 } & & $\mathbf{3 5}$ & $\mathbf{5 0}$ & $\mathbf{8 0}$ & $\mathbf{1 0 0}$ & $\mathbf{1 5 0}$ & $\mathbf{2 0 0}$ & $\mathbf{3 0 0}$ & $\mathbf{5 0 0}$ \\
\hline$b=b_{0}$ & 0.75 & 0.04 & 0.07 & 0.21 & 0.34 & 0.71 & 1.12 & 1.85 & 3.07 \\
& 0.5 & 0.05 & 0.05 & 0.06 & 0.07 & 0.12 & 0.22 & 0.44 & 1.02 \\
& 0.25 & 0.23 & 0.27 & 0.32 & 0.33 & 0.61 & 0.33 & 0.33 & 0.37 \\
$b=0.75 b_{0}$ & 0.125 & 0.55 & 0.68 & 0.85 & 0.91 & 0.94 & 0.98 & 0.93 & 0.73 \\
& 0.75 & 0.04 & 0.05 & 0.12 & 0.19 & 0.39 & 0.65 & 1.11 & 2.09 \\
& 0.5 & 0.07 & 0.08 & 0.10 & 0.11 & 0.17 & 0.27 & 0.46 & 0.93 \\
$b=0.5 b_{0}$ & 0.25 & 0.29 & 0.34 & 0.40 & 0.41 & 0.42 & 0.46 & 0.48 & 0.59 \\
& 0.125 & 0.64 & 0.76 & 0.90 & 0.93 & 0.96 & 1.03 & 1.01 & 1.17 \\
& 0.75 & 0.08 & 0.09 & 0.15 & 0.20 & 0.33 & 0.50 & 0.76 & 1.29 \\
& 0.5 & 0.17 & 0.18 & 0.23 & 0.26 & 0.33 & 0.43 & 0.57 & 0.86 \\
$b=0.25 b_{0}$ & 0.25 & 0.31 & 0.52 & 0.63 & 0.65 & 0.72 & 0.79 & 0.83 & 0.95 \\
& 0.125 & 0.78 & 0.90 & 1.07 & 1.11 & 1.21 & 1.31 & 1.35 & 1.31 \\
& 0.75 & 0.28 & 0.35 & 0.45 & 0.48 & 0.56 & 0.64 & 0.73 & 0.86 \\
& 0.5 & 0.46 & 0.54 & 0.67 & 0.70 & 0.78 & 0.84 & 0.87 & 0.95 \\
& 0.25 & 0.79 & 0.91 & 1.06 & 1.09 & 1.18 & 1.25 & 1.27 & 1.18 \\
& 0.125 & 1.18 & 1.26 & 1.47 & 1.61 & 1.99 & 2.12 & 2.09 & 2.55
\end{tabular}

\section{RESULTS}

To determine the effect of block geometry and dielectric permittivity on the transmit field distribution and efficiency within the spherical phantom, electromagnetic field simulations were performed (Figure 2). The transmit field distribution within the spherical phantom for $d=0.125 b$ was the most consistent regardless of the combination of $b / b_{0}$ and $\varepsilon_{\mathrm{r}}$. Moreover, $d=0.125 b$ (for $b=0.5 b_{0}$ and $b=0.75 b_{0}$ ) resulted in the highest transmit field efficiency in the center of the phantom for all $\varepsilon_{\mathrm{r}}$ values. The most apparent change in the transmit field distribution (two-side lobes and almost no transmit field along the $z$-axis) and decrease in the transmit field efficiency was observed for $d=0.75 b\left(b=b_{0}, b=0.75 b_{0}\right.$, and $\left.b=0.5 b_{0}\right)$ for all $\varepsilon_{\mathrm{r}}$ values (Figure 2). For $d=0.75 b\left(b=0.25 b_{0}\right)$, that effect was still present, but for the higher- $\varepsilon_{\mathrm{r}}$ blocks $\left(\varepsilon_{\mathrm{r}} \geq 200\right)$. A similar change in the transmit field pattern as for $d=0.75 b$ was also observed for thinner blocks with $d=0.5 b$. This was especially prominent for higher $\varepsilon_{\mathrm{r}}\left(\varepsilon_{\mathrm{r}} \geq 150\right)$. Local $\mathrm{SAR}_{10 \mathrm{~g}}$ values for each block are summarized in Table 2. Higher $d / b$ ratios yielded lower $\mathrm{SAR}_{10 g}$ values for narrower blocks $\left(b=0.25 b_{0}\right.$ and $\left.b=0.5 b_{0}\right)$, excluding $d / b=0.75$, for the highest-er values $\left(\varepsilon_{\mathrm{r}} \geq 200\right)$. For wider blocks $\left(b=0.75 b_{0}\right.$ and $\left.b=b_{0}\right)$, SAR $10 \mathrm{~g}$ was the highest for the thickest blocks $(d / b=0.75)$ excluding the lowest $-\varepsilon_{\mathrm{r}}$ values, 35 and 50 (the latter only for $d / b=0.5$ ).

To determine which $\varepsilon_{\mathrm{r}}$ provided the highest transmit field and SAR in the center of the spherical phantom, electromagnetic field simulations were performed using a constant block geometry and dipole antenna length (Figure 3). The electric field distribution was found to depend on $\varepsilon_{\mathrm{r}}$ of the block (Figure 3), and it was significantly different for higher- $\varepsilon_{\mathrm{r}}$ blocks $\left(\varepsilon_{\mathrm{r}} \geq 200\right)$. The highest transmit field efficiency in the center of the phantom was obtained with $\varepsilon_{\mathrm{r}}=300(0.21 \mu \mathrm{T} / \sqrt{ } \mathrm{W})$ and $\varepsilon_{\mathrm{r}}=200$ $(0.197 \mu \mathrm{T} / \sqrt{ } \mathrm{W})$, but at the cost of reduced SAR efficiency $(0.103 \mu \mathrm{T} / \sqrt{ } \mathrm{W} / \mathrm{kg}$ and $0.126 \mu \mathrm{T} / \sqrt{ } \mathrm{W} / \mathrm{kg})$. The highest SAR efficiency in the center was obtained with lower $\varepsilon_{\mathrm{r}}$ values: $0.149 \mu \mathrm{T} / \sqrt{ } \mathrm{W} / \mathrm{kg}$ for $\varepsilon_{\mathrm{r}}=80$, and $0.148 \mu \mathrm{T} / \sqrt{ } \mathrm{W} / \mathrm{kg}$ for $\varepsilon_{\mathrm{r}}=50$. The transmit efficiency (center of the phantom) with $\varepsilon_{\mathrm{r}}=300$ was higher by $33 \%$, and the SAR efficiency was lower by $31 \%$ than with $\varepsilon_{\mathrm{r}}=80$. The transmit efficiency (center of the phantom) with $\varepsilon_{\mathrm{r}}=200$ was higher by $29 \%$, and the SAR efficiency was lower by $15.5 \%$ than with $\varepsilon_{\mathrm{r}}=80$.

To investigate how physical separation between the dielectric block and the cuboid phantom can influence the transmit field pattern and efficiency, an air gap (1-5 mm) between the block and the phantom was assumed for one block geometry ( $\left.b=0.75 b_{0}, d=0.75 b\right)$ for all $\varepsilon_{\mathrm{r}}$ (Figure 4). The larger block was chosen because it produced a highly inefficient transmit field in the spherical phantom (Figure 2, second row). It was found for lower $\varepsilon_{\mathrm{r}}$ values ( 35 and 50 ) that, despite the air gap, the transmit field pattern was very similar to the one obtained with a direct contact between the block and the cuboid phantom, albeit with slightly lower efficiency. For higher $\varepsilon_{\mathrm{r}}$ values $\left(\varepsilon_{\mathrm{r}} \geq 80\right)$, a significant difference in the transmit field pattern and efficiency could be observed. For $\varepsilon_{\mathrm{r}}=80$, the effect was present with a $3-\mathrm{mm}$ air gap, while for higher $\varepsilon_{\mathrm{r}}$ values $\left(\varepsilon_{\mathrm{r}} \geq 200\right)$, a $1-\mathrm{mm}$ air gap was already sufficient, and the observed effect was much more prominent.

The impact of block/phantom physical separation and phantom geometry was further investigated by simulating the y-component of the magnetic field $\left(H_{y}\right)$. Increasing the distance between the phantom and the block by $20 \mathrm{~mm}$ for the two lower$\varepsilon_{\mathrm{r}}(35,50)$ blocks resulted in $H_{y}$ being mostly contained within the blocks (Figure 5A). For higher $\varepsilon_{\mathrm{r}}$ (200), the effect was already prominent with a $0.5-\mathrm{mm}$ air gap, showing that the distance between the block and the phantom for the given $\varepsilon_{\mathrm{r}}$ value significantly influenced the transmit field distribution. The results were compared to those obtained previously for the spherical phantom (5-mm air gap; Figure 5B): $H_{y}$ was mostly contained within the low- $\varepsilon_{\mathrm{r}}$ blocks $\left(\varepsilon_{\mathrm{r}}=35\right.$ and 50$)$ for the spherical phantom, while for the cuboid phantom, $H_{y}$ propagated toward the phantom despite the 5-mm air gap.

To investigate the transmit field distribution within different rectangular block geometries, PMMA boxes were filled with deionized water and imaged by gradient-recalled echo at lowflip angles. Based on the analysis of the simulated transmit field patterns and MR experiments (Figure 6), we interpreted the mode excited in the larger block $(d=0.75 b)$ as $T E_{1 \delta \delta}^{y}$. Using the same approach, we identified the mode in the smaller block $(d=$ $0.25 b)$. Our results indicated that two modes $\left(T E_{11 \delta}^{z}\right.$ and $\left.T E_{1 \delta \delta}^{y}\right)$ were adjacent to each other and could be switched by changing the ratio of the block dimensions ( $d$ in this case).

To understand the impact on an MRI experiment of the different dielectric modes that can be excited by a dielectrically shortened dipole antenna, in vivo studies were performed with one male subject. Two block geometries were investigated: $d=0.25 b, d=0.75 b$. In vivo images of the head, calf, and wrist were obtained (Figure 7). 
A
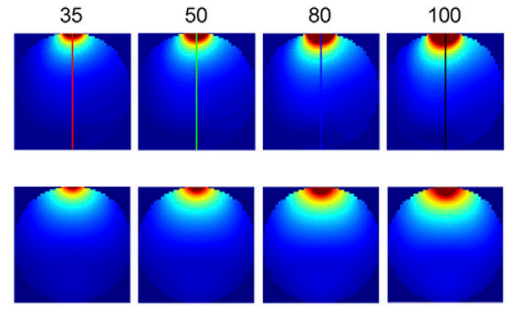

$\varepsilon_{r}$
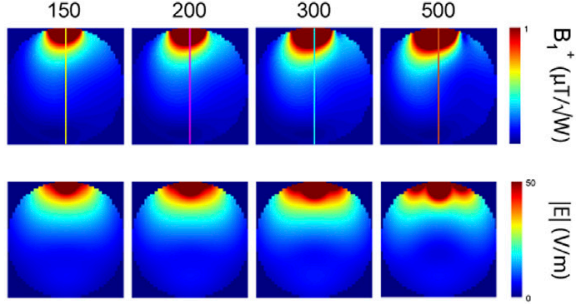

\section{B $\mathrm{B}_{1}{ }^{+}$efficiency $(\mu \mathrm{T} / \sqrt{\mathrm{W}})$}

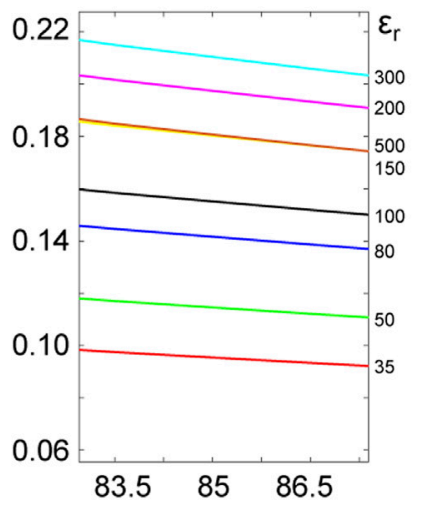

SAR efficiency $(\mu \mathrm{T} / \sqrt{\mathrm{W} / \mathrm{kg}})$

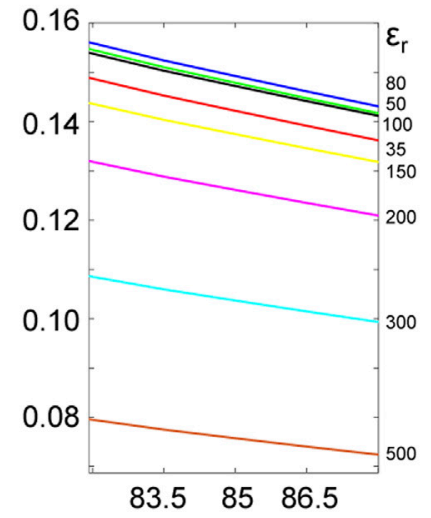

distance $(\mathrm{mm})$

FIGURE 3 | (A) Transmit field and electric field distribution within the spherical phantom for the constant block geometry (160 mm $\times 60 \mathrm{~mm} \times 7.5 \mathrm{~mm})$ and dipole antenna length as a function of dielectric permittivity $\varepsilon_{r}(35,50,80,100,150,200,300$, and 500). The air gap was 5 mm. (B) Transmit and SAR efficiency as functions of distance. The plots were zoomed in ( $85 \mathrm{~mm}$ on the $x$-axis corresponds to the center of the phantom) to better distinguish the differences between the blocks. The highest transmit field efficiency in the center of the phantom was obtained for $\varepsilon_{\mathrm{r}}=300(0.210 \mu \mathrm{T} / \mathrm{JW})$ and $\varepsilon_{\mathrm{r}}=200(0.197 \mu \mathrm{T} / \sqrt{ } \mathrm{W})$ but at the cost of a reduced SAR efficiency $(0.103$ and $0.126 \mu \mathrm{T} / \sqrt{ } \mathrm{W} / \mathrm{kg})$. The highest SAR efficiency in the center was obtained for lower $\varepsilon_{\mathrm{r}}$ values: $0.149 \mu \mathrm{T} / \sqrt{ } \mathrm{W} / \mathrm{kg}$ for $\varepsilon_{\mathrm{r}}=80$ and $0.148 \mu \mathrm{T} / \mathrm{JW} / \mathrm{kg}$ for $\varepsilon_{\mathrm{r}}=50$. Note that in higher- $\varepsilon_{\mathrm{r}}$ blocks $(300,500)$, a higher-order mode $\left(T E_{12 \delta}^{z}\right)$ was excited.

The thinner block provided highly superior image quality for all tissues compared to its thicker counterpart, which yielded very noisy images (very low to no SNR) for the brain, calf, and wrist. However, qualitative differences were apparent among the images obtained using the larger block, and the best image quality was obtained with the wrist, which provided a flatter surface. Significantly, lower image quality was noted for the calf while the lowest was for the head, the body part with the highest level of curvature in this study.

To determine whether the larger block $(160 \mathrm{~mm} \times 70 \mathrm{~mm} \times$ $52.5 \mathrm{~mm}$ ) could still be used as an efficient RF antenna, planewave simulations for six different orientations of the magnetic field vector $\vec{H}$, the electric field vector $\vec{E}$, and the wave number vector $\vec{k}$ were performed (Figure 8). Different mutual orientations of these three vectors led to different magnetic field patterns in the spherical phantom. The mutual orientation of $\vec{H}$ and $\vec{E}: E_{z^{-}} k_{x}$ was consistent with dipole excitation and yielded a transmit field pattern similar to that in Figure 2. There were also other excitation schemes $\left(E_{y}-k_{x}, E_{x}-k_{y}\right.$, and $\left.E_{x}-k_{z}\right)$ that, unlike $E_{z^{-}} k_{x}$, provided efficient magnetic field in the spherical phantom.

\section{DISCUSSION}

This study demonstrates for the first time why certain dipole-fed rectangular dielectric resonator antennas for MRI at $7 \mathrm{~T}$ can preserve (and others do not) the transmit field distribution and efficiency when the dielectric block and the sample are physically separated. We showed that different types of quasi-transverse electric modes, which were induced in the analyzed block geometries by dipole antennas, played the most critical role in this context: $T E_{11 \delta}^{z}$ and $T E_{1 \delta \delta}^{y}$. The approach used in this study constitutes an important extension of prior work [14], which focused on the analysis of the special case where there is a direct contact between a rectangular block and a large cuboid phantom: first, such a condition may not always be realistic: for example, human heads and other body parts have different curvatures, and 


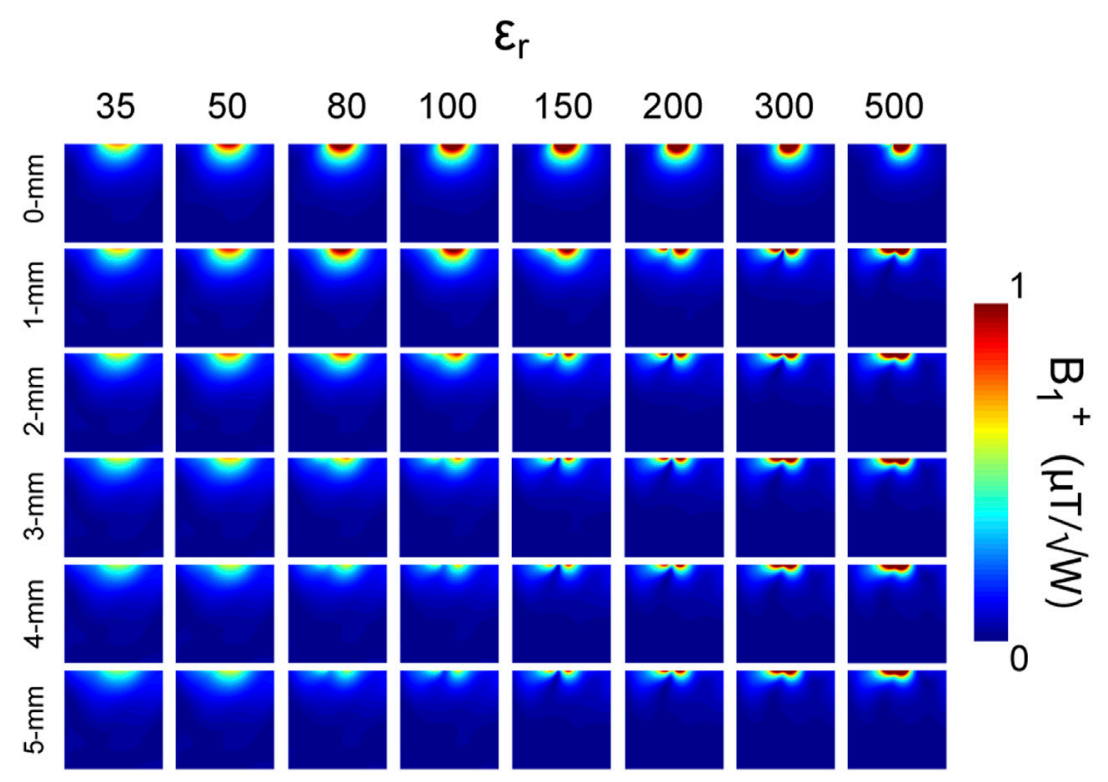

FIGURE 4 | Transmit field distribution in the cuboid phantom as a function of dielectric permittivity of the block and the block/phantom physical separation. The geometry of one of the larger blocks was chosen ( $b=0.75 b_{0}, d / b=0.75-$ Table 1). The effect of five different sizes of an air gap (1, 2, 3, 4, and $\left.5 \mathrm{~mm}\right)$ was investigated for all $\varepsilon_{\mathrm{r}}$ values $(35,50,80,100,150,200,300$, and 500$)$. No significant change was noted in the transmit field pattern for lower $\varepsilon_{\mathrm{r}}$ values (35, 50) when the antenna was moved $5 \mathrm{~mm}$ away from the phantom. The transmit field changed significantly for higher $\varepsilon_{\mathrm{r}}$ values. The higher the $\varepsilon_{\mathrm{r}}$ value of the block, the lower the "threshold" size of the air gap, for which the transmit field pattern changed along with a decreased efficiency.

therefore, a perfect direct contact between the body and a rectangular block is difficult, if not impossible, to achieve in general. Thus, instead of assuming a perfect contact between the dielectric block and phantom, the presence of air gaps is a more realistic consideration.

By using the term "quasi-TE modes", we have referred to Pan et al. [20], who reported on a negligibly weak E-field component which can be observed in the direction of propagation for TE modes in rectangular dielectric resonator antennas. Moreover, the prefix "quasi" refers to a larger group of rectangular geometries which were studied in this work but not optimized to excite "pure" TE modes. In such cases, a nonzero E-field component in the direction of propagation may be observed. The same applies to the distinction between $T E_{11 \delta}^{z}$ and $T E_{1 \delta \delta}^{y}$ modes. These modes can be identified based on the electromagnetic field patterns within a dielectric block (in principle, simulated vector fields are preferable to discern the intricacies between different geometries). To determine $m$, we looked into the magnetic field variation along the $x$-axis (Figure 6, coronal slice), and we found that a full-field maximum is contained within the block (indicating a sharp change in the magnetic field direction at the dielectric boundaries along the $x$-axis), yielding $m=1$ for both blocks. To find $n$, we looked at the magnetic field variation along the $y$-axis (Figure 6, coronal slice). In the case of the $T E_{11 \delta}^{z}$ mode, the field maximum was fully contained within the thinner block, that is, there was a sharp change in the magnetic field direction at the block boundaries along the $y$-axis. This resulted in no magnetic field leaking through the dielectric boundaries along the $y$-axis, and the field decreased to nearly zero at the edge of the block, resulting in $n=1$. In this case, the magnetic field propagates along the $z$-axis through the dielectric wall, and the upper index $z$ was used to better describe this mode. By increasing the dimension $d$ of the block, decreased magnetic field variation along the $y$-axis can be observed, due to an increased contribution of the $T E_{10 \delta}^{y}$ mode ( $n=0$ stands for no magnetic field variation in the $y$-direction), and a similar magnetic field pattern is found in rectangular waveguides for the $T E_{10}$ mode. In the case of the $T E_{10 \delta}^{y}$ mode, the magnetic field is not contained within the block along the $y$-axis, that is, there is no change of magnetic field direction at the boundaries, and the magnetic field leaks through the dielectric walls in both directions along the $y$-axis and propagates down toward the sample. The upper index $y$ therefore serves to indicate the change in the direction of magnetic field propagation for thicker blocks. This change of magnetic field pattern between thinner block and its thicker counterpart is similar to the transition between $T E_{11}$ and $T E_{10}$ in rectangular waveguides (see the subchapter "Rectangular waveguide" in "Transmission lines and waveguides" in Pozar's [19] book). In this work, different, semi-arbitrarily chosen rectangular geometries were analyzed, and $T E_{10 \delta}^{y}$ is considered to be a subcomponent of a larger group of $T E_{1 \delta \delta}^{y}$ modes. We have introduced index $\delta$ to indicate that certain modes have insufficient purity to be interpreted as $T E_{10 \delta}^{y}$ and contributions from both modes $T E_{11 \delta}^{z}$ and $T E_{10 \delta}^{y}$ can be very apparent (see Figure 1). None of the blocks analyzed in this study had index $l$ equal to 1; however, it is clear (Figure 6, axial slice) that a fraction of magnetic field variation can be observed along the $z$-axis for both blocks, and we therefore assigned $l=\delta$ for both blocks.

Dipole-fed rectangular dielectric resonator antennas, in which the $T E_{11 \delta}^{z}$ mode was excited, preserved their transmit field distribution and efficiency regardless of (a) any block/sample physical separation and (b) any level of curvature of the sample (for both phantom and 

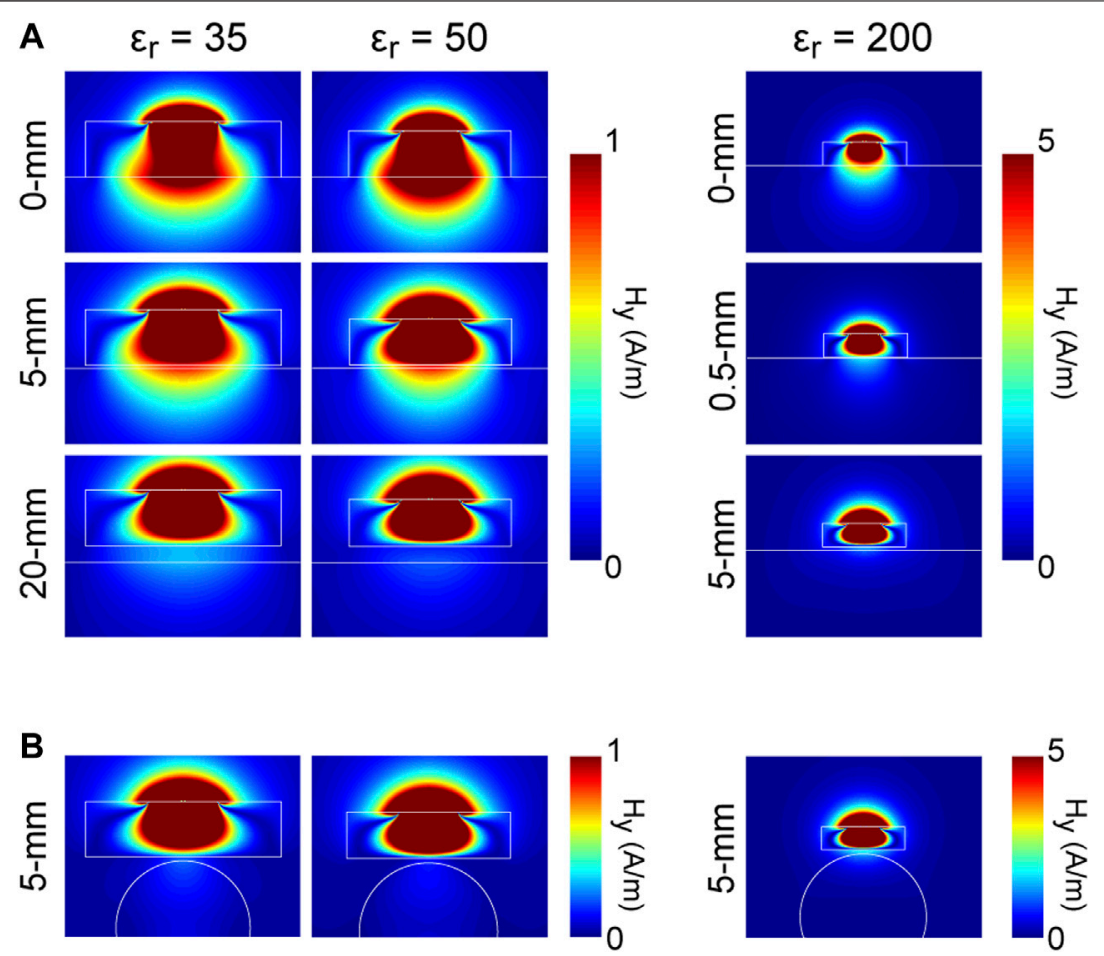

FIGURE 5 | (A) The distribution of the $y$-component (in $x-z$ plane) of the magnetic field $\left(H_{y}\right)$ as a function of dielectric permittivity, phantom geometry, and block/ phantom physical separation. White lines depict the boundaries of the blocks and the phantoms. For the direct contact case (cuboid phantom) as well as for smaller air gap $(5-\mathrm{mm}), H_{y}$ for lower- $\varepsilon_{r}$ blocks $(35,50)$ coupled well to the phantom enabling the propagation of $H$-field in the $z$-direction. For the cuboid phantom (20-mm air gap) and lower- $\varepsilon_{r}$ blocks, $H_{y}$ was mostly confined within the blocks, especially for $\varepsilon_{r}=50$. In the case of $\varepsilon_{r}=200$, a similar effect was observed even with a 0.5 -mm air gap. (B) $H_{y}$ distribution in the cuboid phantom (5-mm air gap) was benchmarked against $H_{y}$ distribution in the spherical phantom (5-mm air gap). The results showed that $H_{y}$ distribution within the block (and therefore the transmit field efficiency) was significantly dependent on the loading geometry.

in vivo experiments). Rectangular dielectric resonator antennas, in which the $T E_{1 \delta \delta}^{y}$ mode was induced, performed poorly when the block was separated from the sample: a significantly altered transmit field distribution and low efficiency was observed. When $T E_{1 \delta \delta}^{y}$ was excited, there were two general cases in which it produced an efficient transmit field: (a) a direct contact with the cuboid phantom (flat surface) regardless of the $\varepsilon_{\mathrm{r}}$ value of the block and (b) a fairly small block/phantom separation but only for low- $\varepsilon_{\mathrm{r}}$ blocks $(35,50)$. Here, condition (b) remains valid as long as the surface of the sample is flat: the resulting transmit field will be a function of the level of curvature of the sample, and it can substantially change when the surface approaches a rounded one (Figure 5B). In vivo experiments showed that the $T E_{1 \delta \delta}^{y}$ mode, unlike $T E_{11 \delta}^{z}$, led to substantially degraded image quality, highlighting the influence of dielectric block geometry and propagation of dielectric modes on the performance of dielectrically shortened dipole antennas. Further discussion on how different parameters can influence the antenna performance is given below.

The propagation of quasi-transverse dielectric modes depends mainly on the ratio of the block dimensions (Figure 1): $a, b$, and $d$ (if the feeding type did not change; Figure 2) and on the feeding type (if the dimensions were constant; Figure 8). Our results show that when $d$ was sufficiently smaller than $b$, a quasi- $T E^{z}$ mode was expected to propagate. However, when dimension $d$ approaches $b$ (even for $d=0.5 b$ ), the dominant mode shifts toward a quasi-TE mode (Figure 2). The main dielectric modes observed in our experiments were interpreted as $T E_{11 \delta}^{z}$ (MR efficient) and $T E_{1 \delta \delta}^{y}$ (MR inefficient; Figure 6). There was a striking difference in performance between these modes, which had a major impact on in vivo experiments (Figure 7). This observation differs from the work of Ipek et al. [14], who reported only one type of mode $\left(T E_{1 \delta 1}^{y}\right)$ : this was possible due to the substantially different boundary conditions (perfect direct contact between the block and the cuboid phantom) in their experiments, leading to the situation in which electromagnetic wave is guided across different dielectric media (no high-/low- $\varepsilon_{\mathrm{r}}$ interface and less reflection). However, for the geometries studied here, $T E_{1 \delta 1}^{y}$ was not allowed to propagate (only for the geometries with $d / b=0.75$, the cutoff frequency was very close to $297.2 \mathrm{MHz}$ (see Eq. 1).

We found that a relatively small $d / b$ ratio $(d=0.5 b$ and $d=0.75 b$; for $\varepsilon_{\mathrm{r}}=500$ even when $d=0.25 b$ ) results in an inefficient transmit field in the spherical phantom (Figure 2): high- $\varepsilon_{\mathrm{r}}$ blocks $\left(\varepsilon_{\mathrm{r}} \geq 200\right)$ can produce an inefficient transmit field for much smaller $d / b$ ratios than low- $\varepsilon_{\mathrm{r}}$ blocks $(35,50)$. This indicates that $T E_{1 \delta \delta}^{y}$ can be excited using smaller a/b/d ratios with higher $\varepsilon_{\mathrm{r}}$ values, and block dimensions optimized for low $\varepsilon_{\mathrm{r}}$ values should not be linearly scaled for high- $\varepsilon_{\mathrm{r}}$ blocks because an inefficient dielectric mode can become more prominent, compromising the antenna performance. Using very "thin" blocks $(d=0.125 b)$ can be advantageous in the context of 


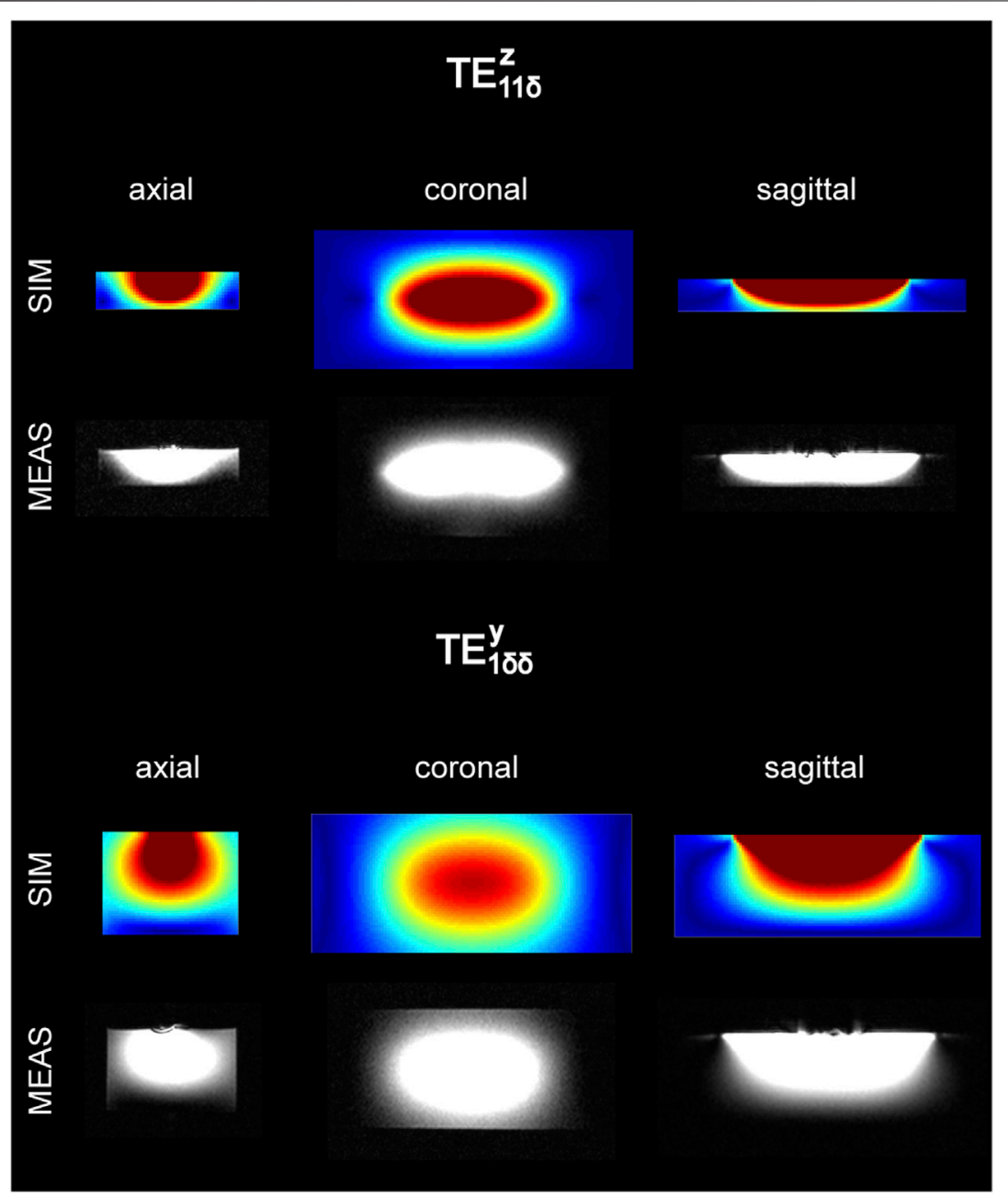

FIGURE 6 | Visualization of dielectric modes: the comparison between the electromagnetic field simulations and magnetic resonance measurements for two elements: thinner one $(d=0.25 b)$ and thicker one $(d=0.75 b)$. The polymethylmethacrylate boxes were filled with deionized water, and their volumes were matched to the dimensions of the dielectric blocks described in the Methods section. Low-flip-angle gradient echo imaging was used. The simulations are in an excellent agreement with the measurements and show significantly different magnetic field distribution between the blocks. The mode that propagates within the thinner block was interpreted as $T E_{11 \delta}^{z}$ and within the thicker one as $T E_{1 \delta \delta}^{y}$. SIM stands for "Simulation" and MEAS for "Measurement".

transmit efficiency (Figure 2) in contrast to the previous report in which $d=b$ for the case of a direct contact between the block and the cuboid phantom [14]. This is possible because the dimension $d$ does not play a key role in excitation of the $T E_{11 \delta}^{z}$ mode and can be significantly reduced. This finding can have important practical implication due to substantial space constraints within the MRI scanner bore and the general need for the "miniaturization" of dielectric blocks.

Note, however, that decreasing $d$ can result in needing larger inductors to tune the antenna, which has an impact on the transmit field efficiency. Thinner blocks are also expected to yield higher SAR values than their thicker counterparts, excluding the wider blocks with $b=b_{0}$ and $b=0.75 b_{0}$, especially for higher $\varepsilon_{\mathrm{r}}$ values $\left(\varepsilon_{\mathrm{r}} \geq 200\right)$. Therefore, certain tradeoffs would have to be considered and accepted depending on the application. If the block dimension $d$ is properly chosen for a given $a$ and $b$, the latter two play the key role in overall antenna performance. With $d=0.125 b$, the most efficient transmit field was observed with $b=0.5 b_{0}$ and $b=0.75 b_{0}$ (for $d=0.125 b)$ for all $\varepsilon_{\mathrm{r}}$ values. The transmit field efficiency for $b=0.5 b_{0}$ was slightly higher $(\sim 2.5 \%)$ than $b=0.75 b_{0}$. However, on average, $20 \%$ higher inductance was needed to tune the antennas with $b=$ $0.5 b_{0}$. Losses associated with higher inductance were not included in the simulations, and they are expected to affect the transmit field efficiency. Moreover, the SAR efficiency for $b=0.5 b_{0}$ was $\sim 7.2 \%$ lower than that for $b=0.75 b_{0}$. Interestingly, according to Eq. 1, the cutoff frequency for the $T E_{11 \delta}^{z}$ mode was almost identical to the resonance frequency of protons at $7.0 \mathrm{~T}$ for blocks with $0.75 b_{0}$.

Transmit field patterns for $d=0.75 b$ (Figure 2) should not be confused with the pattern that would be expected for a standalone loop coil at 297.2 MHz. A transmit field pattern similar to the one for the loop coil can be obtained by replacing the dipole-with a loop-type excitation (see $E_{y}-k_{x}$ excitation from Figure 8). Note that for the ratio $d=0.75 b$, the $T E^{z}$ mode turns into the $T E^{y}$ mode. The upper index indicates that the magnetic field propagates across the dielectric 


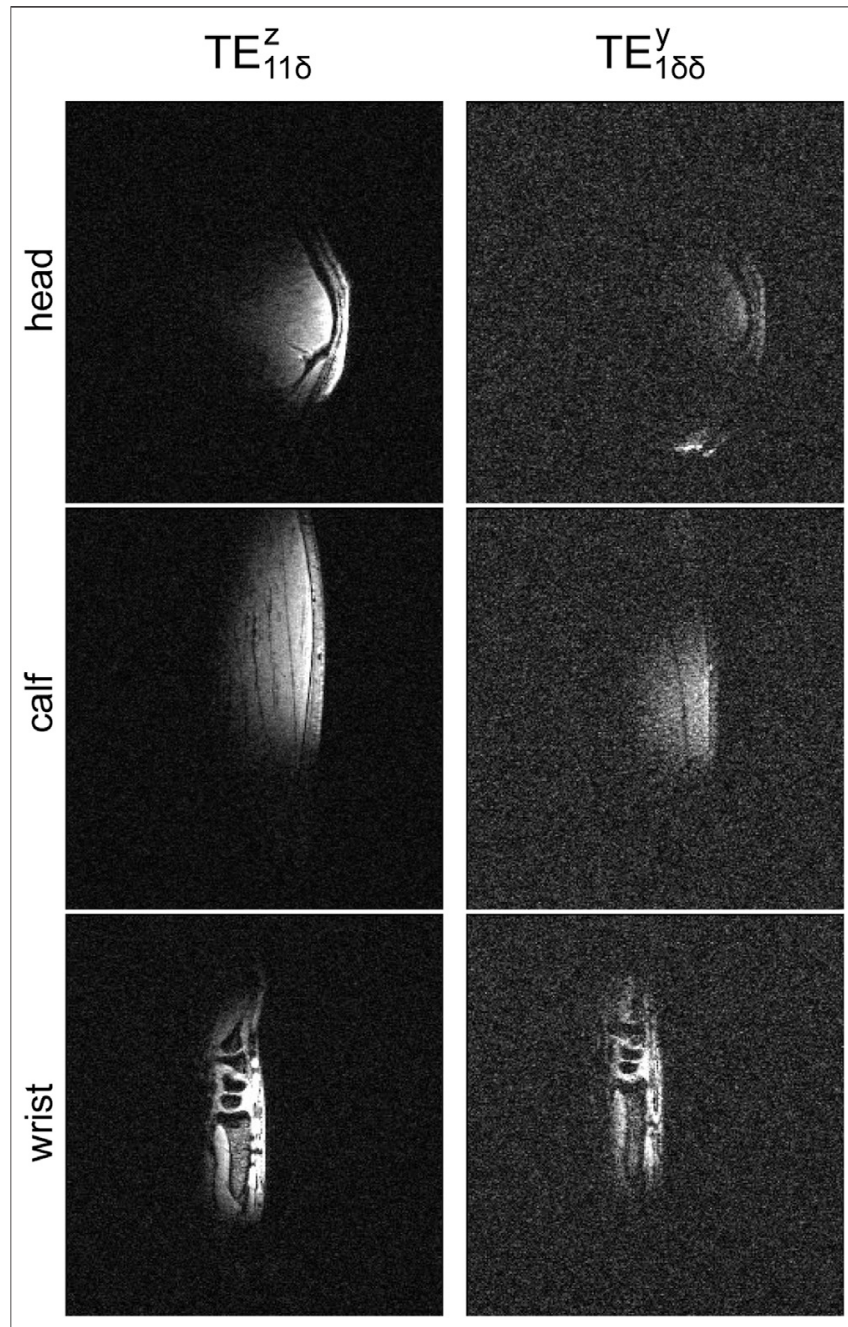

FIGURE 7 | In vivo MRI experiments in one human male subject using two blocks: thinner $(d / b=0.25)$ and thicker one $(d / b=0.75)$. Three different regions of interest (head, calf, and wrist) were investigated. The quality of all the images was significantly compromised for the larger block (very noisy).

The images obtained using the smaller block were superior, and all the tissues can be clearly delineated. Even though the quality of images (larger block) was very low, it can be observed that the quality of the image of the wrist (low level of curvature and almost flat surface) was the highest. The worst quality was noticed for the image of the head (high level of curvature). These results can be benchmarked against the ones obtained for the cuboid (wrist, Figure 5) and the spherical phantom (head, Figure 5). The images have the same absolute intensity.

boundaries along the $y$-axis (almost no propagation along the $z$-axis; see Figure 6, axial view) and partially couples to the phantom placed below the block (the resulting transmit field will also strongly depend on the loading geometry; Figure 7). This applies to all the $\varepsilon_{\mathrm{r}}$ values $(d=0.75 b)$ from Figure 2. The reason why the transmit field pattern for $d=0.75 b$ in Figure 2 appears to be different for different $\varepsilon_{\mathrm{r}}$ values is due to the colorbar with a maximum value of $1 \mu \mathrm{T} / \sqrt{ } \mathrm{W}$ chosen as the maximum value. For higher- $\varepsilon_{\mathrm{r}}$ values, the magnetic field concentration is higher within and in the vicinity of the block, resulting in a substantially higher transmit field in the peripheral regions of the phantom (note that we used a different scale for the simulations for $\varepsilon_{\mathrm{r}}=200$ in Figure 5). In the case of thinner blocks (e.g., $d=0.125 b$ ), the transmit field pattern in the phantom can be considered similar to the one for a standalone dipole antenna. However, in the blocks with $d=0.125 b$, according to Eq. 1, the $T E_{11 \delta}$ mode can still be excited, and the electromagnetic field pattern within the block for $d=0.125 b$ looks very similar to the one for $d=0.25 b$ (see Figure 6, axial view).

The above considerations provide additional evidence why dielectric modes are critical in dielectrically shortened dipole antennas, given the fact that the particular $b / b_{0}$ and $d / b$ ratio increments used in our study were chosen in an arbitrary fashion. Our data also suggest that the propagation of dielectric modes can become a limiting factor in miniaturization of rectangular dielectric blocks in UHF-MRI. We observed that a more efficient transmit field for dielectrically shortened dipole antennas can be achieved when, for a given geometry and $\varepsilon_{\mathrm{r}}$, the cutoff frequency for the $T E_{11 \delta}^{z}$ mode was lower (or close to) the Larmor's frequency.

The effect of the dielectric permittivity of the block on the transmit field performance was analyzed by keeping the distance between the block and the spherical phantom constant $(5 \mathrm{~mm})$ along with the geometry of the block ( $a / b / d$ ratio) and dipole antenna (wire) length (Figure 3). The geometry was chosen such that it could be used as a building block of an 8-channel array [11]. Our data showed that as expected for higher $\varepsilon_{\mathrm{r}}$ values, the electromagnetic field is more concentrated near the block [23]. This led to higher transmit efficiency in the periphery for higher- $\varepsilon_{\mathrm{r}}$ blocks (especially for $\varepsilon_{\mathrm{r}}=300$ and 500, in which the $T E_{12 \delta}^{z}$ mode was excited). However, the best performance in terms of transmit field efficiency among all the analyzed permittivity values in the center of the spherical phantom was found for $\varepsilon_{\mathrm{r}}=300$ and 200, which are $\sim 3 \times$ and $\sim 2 \times$ higher than those reported for the case of a direct contact between the block and the cuboid phantom [14]. This increase, however, was accompanied by a decreased SAR efficiency with higher $\varepsilon_{\mathrm{r}}$ values (Figure 3 ). The block with the best overall performance would therefore represent a trade-off between transmit and SAR efficiency. For example, with $\varepsilon_{\mathrm{r}}=200$, the transmit efficiency was higher by $29 \%$ and the SAR efficiency was lower by $15.5 \%$ than with $\varepsilon_{\mathrm{r}}=80$. The observed SAR increase for $\varepsilon_{\mathrm{r}} \geq 300$ is associated with the higher-order $T E_{12 \delta}^{z}$ mode. This mode had a critical impact on the E-field pattern which was found in the spherical phantom (Figure 3). The lowest transmit efficiency was observed for the lowest $\varepsilon_{\mathrm{r}}$ values $(35,50)$. Note that these geometries were too small to allow propagation of efficient dielectric modes, indicating that dielectric modes play a critical role in the transmit field efficiency of dielectrically shortened dipole antennas. We further note that the observed higher transmit efficiency for higher-order modes $\left(T E_{12 \delta}^{z}\right.$ ) has, to our knowledge, not been reported to-date for dielectrically shortened dipole antennas for UHF-MRI, and this aspect could be further investigated in the future.

The electromagnetic field simulations also showed that the larger rectangular dielectric block, which was coupled to the $T E_{1 \delta \delta}^{y}$ mode using dipole feed placed on the top, could be still used as an efficient RF antenna (Figure 8). One way to couple to a different mode is to change the geometry of the block, and another solution is to change the feeding type. By conducting plane-wave simulations, we found other possible excitation schemes (different mutual orientations of the vectors $\vec{H}, \vec{E}$, and $\vec{k}$ ) that provided an efficient transmit field without any geometrical modifications of the block. Different modes could 


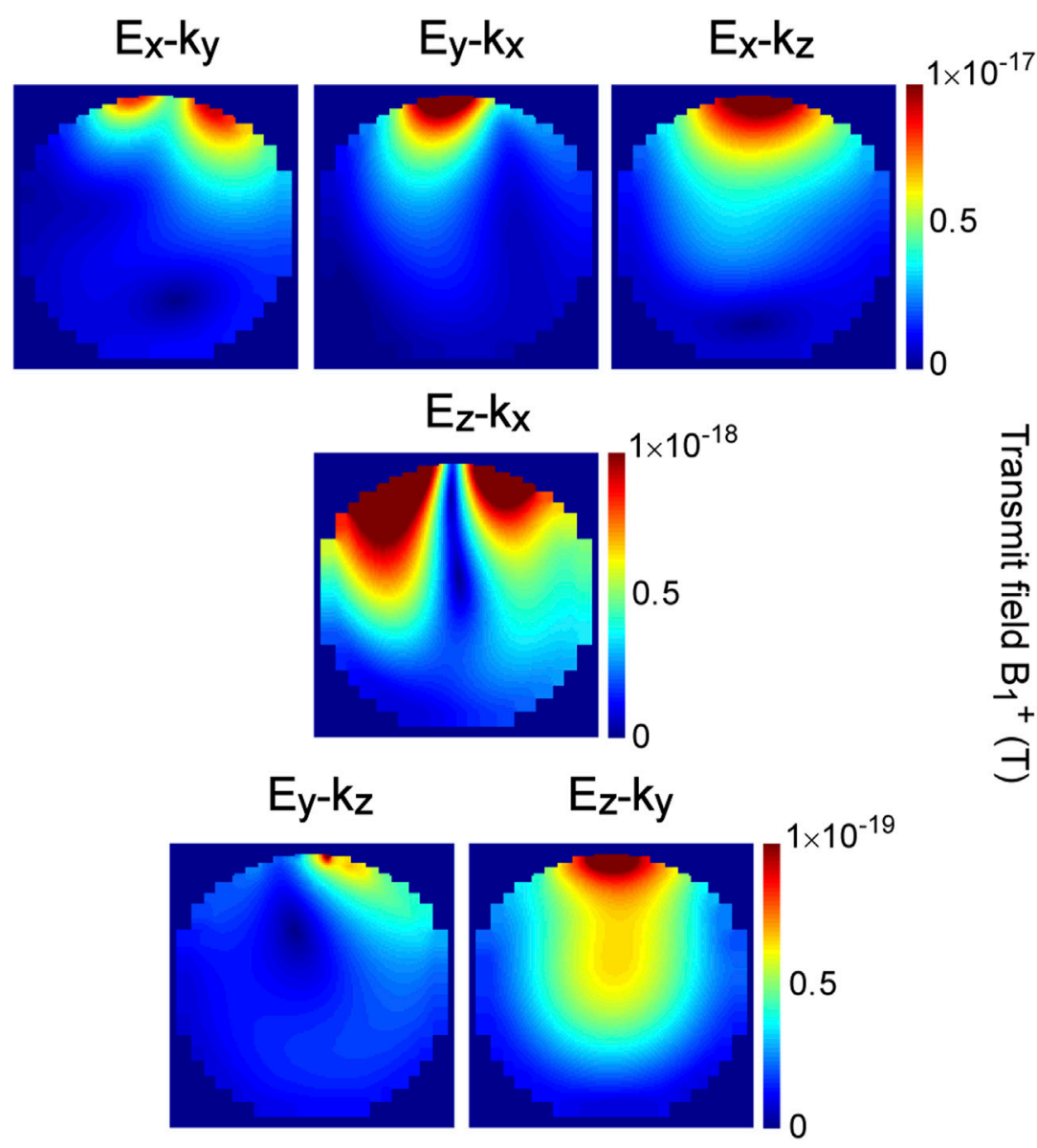

FIGURE 8|Plane-wave simulations: the transmit field distribution in the spherical phantom (5-mm air gap) for one dielectrically shortened dipole antenna ( $\varepsilon_{r}=80$, $160 \mathrm{~mm} \times 70 \mathrm{~mm} \times 52.5 \mathrm{~mm})$. Six different transmit field patterns arise from six different orientations of the $\vec{H}, \vec{E}$, and $\vec{k}$ vectors: $E_{x}-k_{y}, E_{x}-k_{z}, E_{y}-k_{x}, E_{y}-k_{z}, E_{z}-k_{x}$, and $E_{z}-k_{y}$. We see that different coupling schemes can lead to the excitation of different (MR efficient or MR inefficient) dielectric modes for a given dielectric block. Different modes could propagate, for example, by coupling with a small loop coil $\left(E_{y}-k_{x}\right)$, instead of a dipole antenna $\left(E_{z}-k_{x}\right)$. Note that different color scales were assigned to each row of the figure.

propagate for example by coupling with a small loop coil $\left(E_{y}-k_{x}\right)$, instead of a dipole antenna $\left(E_{z}-k_{x} ;\right.$ Figure 8). If a dipole antenna is the desired coupling mechanism, the geometry of the block should be designed according to the guidelines presented in this work, that is, to avoid geometries in which the $T E_{1 \delta \delta}^{y}$ mode could propagate. However, as shown in Figure 8, this does not exclude certain block geometries from being used as an efficient RF antenna. We note that there could be other efficient feed mechanisms (or their combinations, given the fact that some of them are orthogonal) which could be used, for example, $E_{x}-k_{z}$ (Figure 8) and should be investigated in the future [23-28].

The analysis of the transmit field produced within the cuboid phantom by bigger blocks $\left(b=0.75 b_{0}, d=0.75 b\right)$ with $\varepsilon_{\mathrm{r}}$ values ranging from 35 to 500 showed that the distance between the block and the phantom for a given $\varepsilon_{\mathrm{r}}$ is critical for the transmit field pattern and efficiency (Figure 4). For each $\varepsilon_{\mathrm{r}}$ value, five different air gaps (1-5 mm) were investigated, and, despite the air gap, the transmit field pattern for lower $\varepsilon_{\mathrm{r}}$ values ( 35 and 50 ) was very similar to the one obtained with a direct contact between the block and the phantom, albeit with a slightly lower efficiency. For higher $\varepsilon_{\mathrm{r}}$ values $\left(\varepsilon_{\mathrm{r}} \geq 80\right)$, we observed a significant difference in the transmit field pattern and efficiency dependent on the air gap size. For $\varepsilon_{\mathrm{r}}=80$, an altered transmit field pattern along with a decreased efficiency was present with a $3-\mathrm{mm}$ air gap, while for higher $\varepsilon_{\mathrm{r}}$ values $\left(\varepsilon_{\mathrm{r}} \geq 200\right)$ a 1-mm air gap was already sufficient, and the observed effect was much more prominent. This can be explained by the different $\varepsilon_{\mathrm{r}}$ values of the block. The higher the $\varepsilon_{\mathrm{r}}$ value, the higher the concentration of the electromagnetic field within the block and therefore the lower the coupling to the phantom ( $H_{y}$ remains well confined within the block).

Additional simulations demonstrated that a change in the transmit field pattern and efficiency (with $H_{y}$ mostly confined within the block) can be still observed with lower $\varepsilon_{\mathrm{r}}$ values but requires larger air gaps (Figure 5). In general, the lower the $\varepsilon_{\mathrm{r}}$ value, the greater the "threshold" air gap size required to have $H_{y}$ confined within the block and not significantly present in the phantom. In the case of higher $\varepsilon_{\mathrm{r}}$ values (200), the effect was even observed for smaller air gap $(0.5 \mathrm{~mm})$. The latter highlights the importance of this study because the "perfect direct contact" condition seems to be difficult to achieve in practice because even such tiny air gaps can influence antenna performance. Note that even with a direct contact for $\varepsilon_{\mathrm{r}}=500$, a slight change in the transmit field pattern can be already observed 
(Figure 4). The results obtained with the cuboid phantom (5-mm air gap) were compared to the data obtained with the spherical phantom (Figure 5). All the blocks included in the comparison supported the $T E_{1 \delta \delta}^{y}$ mode and produced a very inefficient transmit field along with a substantially altered field pattern in the spherical phantom for each $\varepsilon_{\mathrm{r}}$ value. However, this was not the case for the cuboid phantom for $\varepsilon_{\mathrm{r}}=$ 35 and 50 . This indicates that the geometry of loading can substantially affect block/phantom coupling and influence the transmit field of dielectrically shortened dipole antennas, as discussed further below.

As spherical and rectangular phantom geometries can be considered ideal experimental conditions that may not mimic the practical situation, we performed preliminary in vivo experiments involving one male subject, focusing on three different organs: the head, calf, and wrist (Figure 7). All the investigated anatomical structures had different levels of curvature: human head (high), calf (medium), and wrist (low, almost flat). Dielectric blocks with $d=0.25 b$ and $0.75 b$ were used. All the images obtained when using the thicker block were substantially inferior to the ones when using the thinner block. We observed subtle differences between the images obtained using the thicker block: the image of the wrist had the highest quality, while the image of the head was very noisy, with almost no anatomical details visible, and the calf image quality was intermediate. In vivo data can be compared with our simulations using spherical and cuboid phantoms; the wrist was similar to the flat surface of the cuboid phantom, and the head was obviously more rounded like the spherical phantom. Note that the thicker block used in the in vivo experiments was very close to the optimal design described by Ipek et al. [14], yet its performance was very low. To summarize, when anatomical structures can be considered flat with respect to the bottom surface of the rectangular block, such as the wrist, they can couple better to the antenna's $T E_{1 \delta \delta}^{y}$ mode than organs with curvature such as the head (or even the calf).

An arbitrarily chosen conductivity $\sigma$ (constant for each block) can be considered one of the study's limitations because $\sigma$ is expected to increase with $\varepsilon_{\mathrm{r}}$ of the block. Therefore, the authors recommend to interpret with caution the results concerning transmit efficiency. This, however, is not expected to significantly influence the data obtained in the context of different dielectric modes and transmit field patterns which were affected by them. Also, we would like to point out that expected $\sigma$ increase with $\varepsilon_{\mathrm{r}}$ should have a rather limited impact on future developments, given the fact that there are available technologies which enable manufacturing ceramic blocks with a very high $\varepsilon_{\mathrm{r}}$ value (range of thousands) along with a very low $\sigma$ value of $0.001 \mathrm{~S} / \mathrm{m}$ (roughly 60 times lower than the one used in this study).

In the context of our study, previous work can be divided into two groups: (a) reports in which different, mainly loop-coupled, dielectric structures were used with a clear motivation to induce desired dielectric modes [23-26, 28] and (b) reports in which dielectric structures were used solely for the purpose of shortening dipole antennas $[6,11,13,15,29,30]$. In the case of (a), different types of dielectric modes were investigated, while in the case of (b), even if dielectric modes and their impact on antenna performance were considered by the authors, it was not mentioned in any of those reports. Based on our results, we believe that (a) and (b) should not be considered separately, and this is particularly apparent when the cutoff frequency for a given block geometry and $\varepsilon_{\mathrm{r}}$ is below the NMR frequency. In this study, we showed that dielectric modes play a key role in the antenna's overall performance when the block is separated from the sample. Therefore, by treating such an element as a dipolefed dielectric resonator antenna rather than dielectrically shortened dipole antenna, we highlight the impact of dielectric modes on overall performance of a dipole antenna. This aspect could be further investigated by looking into transmit field patterns produced by combinations of different dipole antenna geometries with different dielectric structures. We have already conducted a study [16], in which we investigated the elements developed by Winter et al. [11], SanchezHeredia et al. [13], and a scaled-version of Raaijmakers et al. [6], and we found that exactly the same types of modes demonstrated in this work were excited.

We conclude that the approach presented in this study can offer guidance and new insights into the design of rectangular dielectric resonator antennas for MRI at $7 \mathrm{~T}$, given the growing number of such antenna designs for UHF-MRI [29-31]. These findings should also be relevant for geometries other than the rectangular ones and for higher Larmor frequencies than the one investigated in this study.

\section{DATA AVAILABILITY STATEMENT}

The original contributions presented in the study are included in the article/Supplementary Material, and further inquiries can be directed to the corresponding author.

\section{ETHICS STATEMENT}

The studies involving human participants were reviewed and approved by the Swiss cantonal ethics committee. The patients/ participants provided their written informed consent to participate in this study. Written informed consent was obtained from the individual(s) for the publication of any potentially identifiable images or data included in this article.

\section{AUTHOR CONTRIBUTIONS}

DW designed the study, performed all of the numerical simulations, designed and built the prototypes used in in vivo experiments, analyzed and interpreted the data, prepared the figures, and wrote the manuscript. RG analyzed and interpreted the data and revised the manuscript.

\section{ACKNOWLEDGMENTS}

The authors wish to thank Hikari Yoshihara (EPFL) for proofreading the manuscript, Lijing Xin (CIBM and EPFL) for helping with in vivo experiments, and Andre Kuehne (MRI.TOOLS, Berlin, Germany) for a helpful discussion. They acknowledge access to the facilities and expertise of the CIBM Center for Biomedical Imaging, a Swiss research center of excellence founded and supported by Lausanne University Hospital (CHUV), University of Lausanne (UNIL), Ecole polytechnique fédérale de Lausanne (EPFL), University of Geneva (UNIGE), and Geneva University Hospitals (HUG). 


\section{REFERENCES}

1. Ladd ME, Bachert P, Meyerspeer M, Moser E, Nagel AM, Norris DG, et al. Pros and Cons of Ultra-high-field MRI/MRS for Human Application. Prog Nucl Magn Reson Spectrosc (2018) 109:1-50. doi:10.1016/j.pnmrs.2018.06.001

2. Roemer PB, Edelstein WA, Hayes CE, Souza SP, and Mueller OM. The NMR Phased Array. Magn Reson Med (1990) 16:192-225. doi:10.1002/mrm. 1910160203

3. Sodickson DK, and Manning WJ. Simultaneous Acquisition of Spatial Harmonics (SMASH): Fast Imaging with Radiofrequency Coil Arrays. Magn Reson Med (1997) 38:591-603. doi:10.1002/mrm.1910380414

4. Pruessmann KP, Weiger M, Scheidegger MB, and Boesiger P. SENSE: Sensitivity Encoding for Fast MRI. Magn Reson Med (1999) 42:952-62. doi:10.1002/(sici)1522-2594(199911)42:5<952:aid-mrm16>3.0.co;2-s

5. Griswold MA, Jakob PM, Heidemann RM, Nittka M, Jellus V, Wang J, et al. Generalized Autocalibrating Partially Parallel Acquisitions (GRAPPA). Magn Reson Med (2002) 47:1202-10. doi:10.1002/mrm.10171

6. Raaijmakers AJE, Ipek O, Klomp DWJ, Possanzini C, Harvey PR, Lagendijk JJW, et al. Design of a Radiative Surface Coil Array Element at 7 T: the SingleSide Adapted Dipole Antenna. Magn Reson Med (2011) 66:1488-97. doi:10. 1002/mrm.22886

7. Raaijmakers AJE, Italiaander M, Voogt IJ, Luijten PR, Hoogduin JM, Klomp DWJ, et al. The Fractionated Dipole Antenna: A New Antenna for Body Imaging at 7 T Esla. Magn Reson Med (2016) 75:1366-74. doi:10.1002/mrm. 25596

8. Ertürk MA, Raaijmakers AJE, Adriany G, Uğurbil K, and Metzger GJ. A 16channel Combined Loop-dipole Transceiver Array for 7 T Esla Body MRI. Magn Reson Med (2017) 77:884-94. doi:10.1002/mrm.26153

9. Clément J, Gruetter R, and Ipek Ö. A Combined 32-channel Receive-loops/8channel Transmit-dipoles Coil Array for Whole-brain MR Imaging at 7T. Magn Reson Med (2019) 82:1229-41. doi:10.1002/mrm.27808

10. Lattanzi R, Wiggins GC, Zhang B, Duan Q, Brown R, and Sodickson DK. Approaching Ultimate Intrinsic Signal-To-Noise Ratio with Loop and Dipole Antennas. Magn Reson Med (2018) 79:1789-803. doi:10.1002/mrm.26803

11. Winter L, Özerdem C, Hoffmann W, Santoro D, Müller A, Waiczies H, et al. Design and Evaluation of a Hybrid Radiofrequency Applicator for Magnetic Resonance Imaging and RF Induced Hyperthermia: Electromagnetic Field Simulations up to 14.0 Tesla and Proof-Of-Concept at 7.0 Tesla. PLoS One (2013) 8:e61661. doi:10.1371/journal.pone.0061661

12. Oezerdem C, Winter L, Graessl A, Paul K, Els A, Weinberger O, et al. 16channel bow Tie Antenna Transceiver Array for Cardiac MR at 7.0 Tesla. Magn Reson Med (2016) 75:2553-65. doi:10.1002/mrm.25840

13. Sanchez-Heredia JD, Avendal J, Bibic A, and Lau BK. Radiative MRI Coil Design Using Parasitic Scatterers: MRI Yagi. IEEE Trans Antennas Propagat (2018) 66:1570-5. doi:10.1109/TAP.2018.2794400

14. Ipek O, Raaijmakers A, Lagendijk J, Luijten P, and van den Berg C, Optimization of the Radiative Antenna for 7-T Magnetic Resonance Body Imaging, Concepts Magn Reson (2013) 43B. p. 1-10. doi:10.1002/cmr.b.21224

15. Eigentler TW, Winter L, Han H, Oberacker E, Kuehne A, Waiczies H, et al. Wideband Self-Grounded Bow-Tie Antenna for Thermal MR. NMR Biomed (2020) 33:33. doi:10.1002/nbm.4274

16. Wenz D, and Gruetter R. Dielectrically-Shortened Dipole Antennas for MRI at 7.0 T: Thick or Thin? Proc Intl Soc Mag Reson Med (2020) 28:4087.

17. Mongia RK, and Bhartia P. Dielectric Resonator Antennas-A Review and General Design Relations for Resonant Frequency and Bandwidth. Int J Microw Mill-Wave Comput-Aided Eng (1994) 4:230-47. doi:10.1002/ mmce. 4570040304
18. Keyrouz S, and Caratelli D. Dielectric Resonator Antennas: Basic Concepts, Design Guidelines, and Recent Developments at Millimeter-Wave Frequencies. Int J Antennas Propagation (2016) 2016:1-20. doi:10.1155/ 2016/6075680

19. Pozar DM. Microwave Engineering. 4th ed. Hoboken, NJ: Wiley (2012).

20. Pan YM, Leung KW, and Lu K. Study of Resonant Modes in Rectangular Dielectric Resonator Antenna Based on Radar Cross Section. IEEE Trans Antennas Propagat (2019) 67:4200-5. doi:10.1109/TAP.2019.2911198

21. Wen H, Jaffer FA, Denison TJ, Duewell S, Chesnick AS, and Balaban RS. The Evaluation of Dielectric Resonators Containing H2O or D2O as RF Coils for High-Field MR Imaging and Spectroscopy. J Magn Reson Ser B (1996) 110: 117-23. doi:10.1006/jmrb.1996.0019

22. Webb AG. Visualization and Characterization of Pure and Coupled Modes in Water-Based Dielectric Resonators on a Human 7T Scanner. J Magn Reson (2012) 216:107-13. doi:10.1016/j.jmr.2012.01.013

23. O'Reilly TPA, Ruytenberg T, and Webb AG. Modular Transmit/receive Arrays Using Very-High Permittivity Dielectric Resonator Antennas: Very-High Permittivity DRA Arrays. Magn Reson Med (2018) 79:1781-8. doi:10.1002/ mrm.26784

24. Aussenhofer SA, and Webb AG. Design and Evaluation of a Detunable Water-Based Quadrature HEM11 Mode Dielectric Resonator as a New Type of Volume Coil for High Field MRI. Magn Reson Med (2012) 68:1325-31. doi:10.1002/mrm.24451

25. Aussenhofer SA, and Webb AG. An Eight-Channel Transmit/receive Array of TE01 Mode High Permittivity Ceramic Resonators for Human Imaging at 7T. J Magn Reson (2014) 243:122-9. doi:10.1016/j.jmr.2014.04.001

26. Ruytenberg T, and Webb AG. Design of a Dielectric Resonator Receive Array at 7 Tesla Using Detunable Ceramic Resonators. J Magn Reson (2017) 284: 94-8. doi:10.1016/j.jmr.2017.09.015

27. Ruytenberg T, O'Reilly TP, and Webb AG. Design and Characterization of Receive-Only Surface Coil Arrays at 3T with Integrated Solid High Permittivity Materials. J Magn Reson (2020) 311:106681. doi:10.1016/j.jmr.2019.106681

28. Moussu MAC, Glybovski SB, Abdeddaim R, Craeye C, Enoch S, Tihon D, et al. Imaging of Two Samples with a Single Transmit/receive Channel Using Coupled Ceramic Resonators for MR Microscopy at 17.2 T. NMR Biomed (2020) 33:e4397. doi:10.1002/nbm.4397

29. Oberacker E, Kuehne A, Oezerdem C, Nadobny J, Weihrauch M, Beck M, et al. Radiofrequency Applicator Concepts for thermal Magnetic Resonance of Brain Tumors at $297 \mathrm{MHz}$ (7.0 Tesla). Int J Hyperthermia (2020) 37: 549-63. doi:10.1080/02656736.2020.1761462

30. Woo MK, DelaBarre L, Lagore R, Jungst S, Yang Q, Zhang B, et al. Improved B1+ Efficiency of a Dipole Antenna Equipped with High Dielectric Constant (HDC) Materials at 10.5T. Proc Intl Soc Mag Reson Med (2019) 27:1498.

31. Sadeghi-Tarakameh A, Jungst S, Wu X, Lanagan M, Adriany G, Metzger GJ, et al. A New Coil Element for Highly-Dense Transmit Arrays: An Introduction to Non-uniform Dielectric Substrate (NODES) Antenna. Proc Intl Soc Mag Reson Med (2019) 27:0732.

Conflict of Interest: The authors declare that the research was conducted in the absence of any commercial or financial relationships that could be construed as a potential conflict of interest.

Copyright (c) 2021 Wenz and Gruetter. This is an open-access article distributed under the terms of the Creative Commons Attribution License (CC BY). The use, distribution or reproduction in other forums is permitted, provided the original author(s) and the copyright owner(s) are credited and that the original publication in this journal is cited, in accordance with accepted academic practice. No use, distribution or reproduction is permitted which does not comply with these terms. 